\title{
Free-convection flow past a horizontal surface in a nanofluid with suction/injection
}

\begin{abstract}
The present work deals with the steady free-convection boundary-layer flow past a horizontal permeable surface embedded in a porous medium filled with a nanofluid. The similarity transformation was applied to the governing equations to transform into a system of ordinary differential equations, which are then solved numerically using Runge-Kutta-Fehlberg fourthfifth order (RKF45) method. Three types of nanoparticles which are titania (TiO2), alumina (A12O3), and copper $(\mathrm{Cu})$ in the based fluid of water are considered to investigate the effect of the nanoparticle volume fraction parameter, $g$. Results are presented and discussed for the local Nusselt number, the surface velocity, the temperature profiles, and the velocity profile. It is found that the imposition of suction/injection has an impact on the velocity profiles and temperature profiles.
\end{abstract}

Keyword: Suction/injection; Free-convection boundary-layer; Nanofluid 